\title{
Linx
}

Revue des linguistes de l'université Paris X Nanterre

5 | 1994

La négation

\section{Le rôle de la sémantique dans l'interprétation de aucun}

Christina Heldner

\section{(2) OpenEdition}

Journals

Édition électronique

URL : http://journals.openedition.org/linx/1217

DOI : $10.4000 /$ linx. 1217

ISSN : 2118-9692

Éditeur

Presses universitaires de Paris Nanterre

\section{Édition imprimée}

Date de publication : 1 juin 1994

Pagination : 255-278

ISSN : 0246-8743

\section{Référence électronique}

Christina Heldner, «Le rôle de la sémantique dans l'interprétation de aucun », Linx [En ligne], 5 | 1994,

mis en ligne le , consulté le 19 avril 2019. URL : http://journals.openedition.org/linx/1217 ; DOI :

10.4000/linx.1217

Ce document a été généré automatiquement le 19 avril 2019.

Département de Sciences du langage, Université Paris Ouest 


\title{
Le rôle de la sémantique dans l'interprétation de aucun
}

\author{
Christina Heldner
}

\section{Introduction}

1 Un phénomène souvent étudié, c'est l'ambiguïté provoquée par la présence, au sein de la même proposition, d'une négation et d'un quantificateur (notamment TOUT, BEAUCOUP ou UN). De nombreux chercheurs ont noté l'absence d'un rapport systématique entre l'ordre superficiel des deux opérateurs et une interprétation donnée. Les descriptions préférées ont consisté à représenter cette ambiguïté sous forme d'interactions de portée à un niveau sémantique sous-jacent. Avec l'indéfini AUCUN - qui fonctionne comme un quantificateur existentiel incorporant une négation - le problème ne se pose pas de la même manière, vu la fusion des deux éléments dans une seule unité lexicale. Néanmoins, l'interprétation des syntagmes nominaux déterminés par AUCUN est susceptible d'une variation qui a peu retenu l'attention des linguistes.

2 Dans un article récent (Heldner, 1992), je présente une analyse selon laquelle les SN en AUCUN reçoivent tantôt une interprétation non référentielle (c'est l'analyse traditionnelle), tantôt une interprétation référentielle. Des arguments sont avancés en faveur de l'hypothèse que la première de ces lectures est à rattacher aux énoncés existentiels et la seconde aux énoncés déclaratifs. ${ }^{1}$ Quant au problème d'identifier les facteurs permettant de sélectionner l'une ou l'autre lecture, il n'est pas abordé dans cet article. En fait, la distinction établie entre SN référentiels et non référentiels repose sur une analyse en termes ensemblistes. Le but que je me propose ici, c'est de dégager quelques-uns des facteurs capables d'influencer l'interprétation de AUCUN. Etant donné l'invariabilité formelle des SN en AUCUN, ceci revient à préciser la distinction entre énoncés existentiels et déclaratifs. 


\section{Interprétation des SN introduits par AUCUN}

3

Mais tout d'abord il me semble utile de rappeler brièvement les caractéristiques des deux lectures de AUCUN. Conformément à l'usage courant, j'entends par énoncé existentiel un énoncé employé pour asserter l'existence ou la non-existence d'objets de la catégorie désignée par le nom tête du SN sujet ou objet. ${ }^{2}$ Il peut s'agir ou bien d'une existence ou d'une non-existence au sens absolu ${ }^{3}$, ou bien d'une présence ou d'une absence localisée dans le temps et l'espace. Dans les énoncés déclaratifs, l'assertion concerne plutôt l'action, le processus ou l'état (etc.) désignés par le prédicat. Suivant qu'il s'agit d'une affirmation ou d'une négation, il est asserté que ce qui est désigné par le prédicat s'applique ou ne s'applique pas au référent du sujet (ou de l'objet), dont l'existence n'est pas assertée, mais présupposée. ${ }^{4}$

4 Une assertion d'existence - on le sait - s'exprime typiquement par une structure impersonnelle du type il existe ou il y a. ${ }^{5}$ Les énoncés de (1) renferment chacun une construction impersonnelle accompagnée d'une négation. Ce qui est asserté, c'est donc la non-existence ou l'absence de quelque chose dans la situation évoquée.

1 a. Là-bas, il n'y a pas de chaise roulante. Les handicapés sont assis par terre, et ils se déplacent à la force de leurs poignets. (Libre Belgique, 860216)

b. Il n'y a donc eu aucune évaporation de richesses, mais simplement transfert de la poche des perdants dans la poche des gagnants. (Libre Belgique, 871128)

c. Il n'existe pas de police d'assurance devant l'évolution historique... (Le Soir, 880402)

d. Et il est vrai qu'il n'existe aucun texte légal fixant les compétences des personnes chargées de la supervision des stages. (Libre Belgique, 860213)

Un fait sans doute moins remarqué, c'est qu'une assertion de non-existence peut également se faire au moyen d'autres constructions, comme par exemple celles de (2), qui font figurer un SN introduit par AUCUN :

2 a. Aucun souffle d'air ne passait dans la brume épaisse endormie sur le fleuve. (Le

Soir, 880413)

b. Aucun cas d'intoxication n'a pour l'instant été enregistré en Suède. (Libre Belgique, 871127)

c. Aucun contrôle ne sera effectué ce printemps à l'usine d'aluminium de Martigny. (

La Tribune de Genève, 880303)

Clairement, ce qui est asserté par (2a), c'est l'absence de souffles d'air dans la brume endormie sur le fleuve. Il s'agit, autrement dit, d'un énoncé véhiculant une proposition existentielle. Observons au passage l'impossibilité d'une lecture signifiant qu'il y avait des souffles d'air dans la situation évoquée, mais que ceux-ci restaient immobiles. Ceci veut dire que (2a) rejette une interprétation «déclarative». Semblablement, (b) et (c) assertent la non-existence de cas d'intoxication et d'opérations de contrôle. On constate ainsi que, dans tous ces exemples, le SN sujet n'a pas de référent en ce sens qu'il ne renvoie à aucun segment de réalité. L'ensemble visé par le nom tête est donc vide au lieu et au temps indiqués. C'est pourquoi je parlerai à leur sujet de SN non référentiels. Notons par ailleurs que ces énoncés acceptent une paraphrase impersonnelle :

3 a. Il ne passait aucun souffle d'air dans la brume épaisse endormie sur le fleuve.

b. Pour l'instant, il n'a été enregistré aucun cas d'intoxication en Suède.

c. Il ne sera effectué aucun contrôle ce printemps à l'usine d'aluminium de Martigny.

Dans les énoncés déclaratifs, les SN déterminés par AUCUN jouent un rôle différent. Considérons d'abord quelques exemples : 
4 a. Aucune vitre ou vitrine n'a résisté dans un rayon de 100 mètres autour du lieu de l'explosion. (Libre Belgique, 860929)

b. Aucun logement social n'a été rénové ou amélioré ces trois dernières années [...] (Le Soir, 880412)

c. Les conditions étaient idéales et aucun joueur ne rechignait à la tâche. (La Tribune de Genève, 880229)

d. Aucune équipe ne s'impose vraiment dans l'un quelconque des sept groupes [...] ( Libre Belgique, 860214)

De toute évidence, (4a) n'a pas été émis pour établir l'absence de vitres et de vitrines dans un rayon de 100 mètres autour du lieu de l'explosion. Au contraire, l'existence d'un nombre défini de vitres spécifiques est présupposée par le locuteur. Ce qui est asserté, c'est que les vitres en question se sont toutes brisées. En fait, (4a) semble proche de (5), qui fait apparaître un SN défini en position de sujet:

5 Les vitres et les vitrines n'ont pas résisté dans un rayon de 100 mètres autour du lieu de l'explosion.

Ainsi je soutiendrai que des SN comme aucune vitre ou vitrine, dans (4a), sont référentiels au même titre que les vitres et les vitrines dans (5). Sémantiquement, l'énoncé (5) se différencie de (4a) surtout par le fait qu'il entraîne une interprétation collective: il asserte au sujet des membres d'un certain sous-ensemble de vitres, pris globalement, que le prédicat AVOIR RESISTE ne s'y applique pas. Ce qui est asserté par (4a), qui n'admet qu'une interprétation distributive, est plutôt ceci : quelle que soit la vitre envisagée, le prédicat AVOIR RESISTE ne s'y applique pas. En tant que quantificateur existentiel, l'indéfini AUCUN opère donc ici sur un sous-ensemble de vitres dont la présence est présupposée, et non pas sur l'ensemble virtuel de toutes les vitres. S'il ne sélectionne aucun de ses membres, c'est qu'il incorpore aussi une négation. A cet égard, il se comporte comme avec les énoncés de (2). La différence cruciale entre les énoncés du type de (4) et ceux du type de (2), c'est donc ce sous-ensemble d'objets dont la présence est présupposée dans la situation d'énonciation. Une analyse analogue peut être proposée pour les exemples $(4 \mathrm{~b}-\mathrm{d})$. La figure 1 représente une tentative de visualiser schématiquement les aspects pertinents des deux types de quantification intervenant dans les énoncés existentiels et déclaratifs.

Figure 1

ENONCES DECLARATIFS

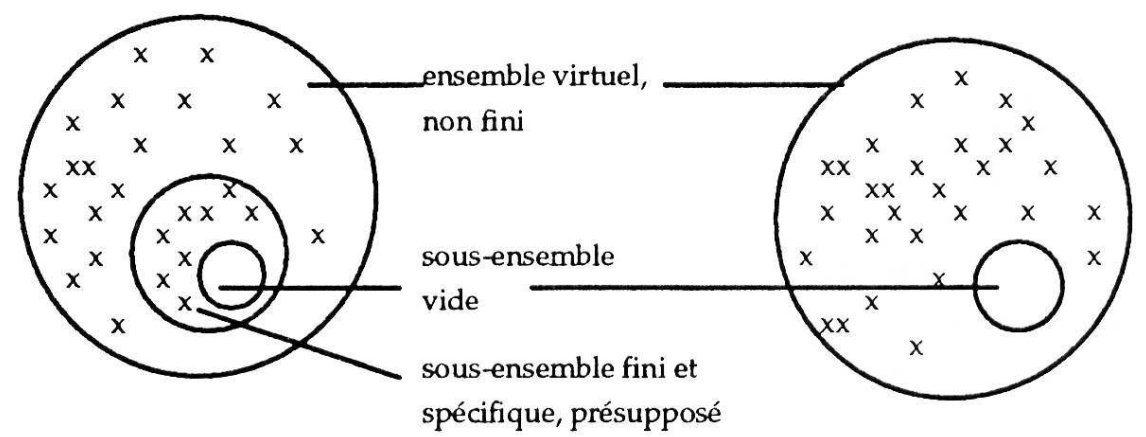

Aucun plancher n'a cédé.

\section{ENONCES EXISTENTIELS}

Aucune arme n'a été trouvé

en la possession des deux voleurs.

Vu la référentialité des SN en AUCUN figurant dans un énoncé déclaratif, on ne s’étonne 
pas de constater qu'ils autorisent une paraphrase par AUCUN DES N sans subir un changement de sens notable. L'effet produit par cette paraphrase, c'est justement une explication du caractère référentiel du SN en question :

6 a. Aucune des vitres ou vitrines n'a résisté dans un rayon de 100 mètres autour du lieu de l'explosion.

b. Aucun des logements sociaux n'a été rénové ou amélioré ces trois dernières années.

c. Aucun des joueurs ne rechignait à la tâche.

d. Aucune des équipes ne s'impose vraiment dans l'un quelconque des sept groupes.

Rien de tel pour les énoncés existentiels. D’une façon générale, ceux-ci acceptent mal cette paraphrase. Ainsi on obtient une modification du sens incompatible avec le contexte discursif en substituant AUCUN DES N à AUCUN N dans les exemples de (2), fait qui révèle la non référentialité du SN sujet.

7 a. Aucun des souffles d'air ne passait dans la brume épaisse endormie sur le fleuve.

b. Aucun des cas d'intoxication n'a pour l'instant été enregistré en Suède.

c. Aucun des contrôles ne sera effectué ce printemps à l'usine d'aluminium de Martigny.

Dans certains cas, la paraphrase par AUCUN DES N produit même un résultat douteux. Que l'on compare (a) et (b) de (8) :

8 a. Qui est d'accord pour ce projet, qui ne prévoit aucune place de parking? (Le Soir, 880406)

b. ? Qui est d'accord pour ce projet, qui ne prévoit aucune des places de parking?

Inversement, les énoncés déclaratifs semblent rejeter une para-phrase impersonnelle. En témoignent les exemples (a), (c) et (d) de (9) formés à partir de (4a-d) :

9 a. * Il n'a résisté aucune vitre ou vitrine dans un rayon de 100 mètres...

b. Il n'a été rénové ou amélioré aucun logement social ces trois dernières années.

c. * Il ne rechignait aucun joueur à la tâche.

d. * Il ne s'impose vraiment aucune équipe dans l'un quelconque des sept groupes.

Or, (9b) est acceptable. En supposant que la construction impersonnelle soit en principe interdite dans les contextes déclaratifs, on pourrait songer à proposer l'hypothèse suivante pour rendre compte du compor-tement de (4b): le refus de la paraphrase impersonnelle présuppose un énoncé univoquement déclaratif. En effet, contrairement à (4a), (4c) et (4d), (4b) accepte une lecture existentielle à côté de la lecture déclarative, du moins hors de son contexte.

\section{Points de départ méthodologiques}

Nous venons de le constater : les SN introduits par AUCUN s'ouvrent tantôt à une lecture référentielle, tantôt à une lecture non référentielle. Si la définition de ce qu'on entend par énoncé existentiel est relativement claire et bien établie, il n'en va pas de même pour la notion d'énoncé déclaratif. Au niveau de l'extension, nous ne disposons pas de critères formels permettant de délimiter avec sûreté quels énoncés concrets sont existentiels et lesquels sont déclaratifs (abstraction faite des constructions impersonnelles en il y a et il existe). La question se pose donc de savoir quels facteurs déterminent l'interprétation d'un énoncé donné à cet égard. La connaissance de ces facteurs permettrait en même temps de prévoir dans quelles conditions les énoncés hébergeant un SN en AUCUN manifesteront une ambiguïé. 
15 En ce qui concerne l'utilité de cette distinction, signalons qu'un premier motif pour la maintenir est lié aux problèmes posés par un traitement automatique du français. Une description de ce phénomène s'impose notamment si l'on veut construire un analyseur conversationnel qui simule le comportement humain au niveau de la compréhension. Tant que celui-ci ne sera pas en mesure d'identifier les référents introduits dans le discours, il sera incapable de faire des inférences valables à partir des données qu'on lui présente. Un deuxième motif se rapporte à la traduction automatisée ou assistée par machine. En effet, il existe des langues - par exemple les langues scandinaves et le russe dans lesquelles la distinction entre phrases existentielles et déclaratives se manifeste ouvertement dans la forme du syntagme nominal correspondant à AUCUN N ${ }^{6}$

Comment procéder pour découvrir ces facteurs? A mon avis, un corpus constitue un instrument heuristique important pour quiconque voudrait aborder un problème de ce genre. Pour avoir une idée générale de l'emploi de AUCUN, j'ai donc commencé par consulter deux corpus informatisés, représentant ensemble environ deux millions de mots courants. Le premier se compose de journaux, le second est littéraire. ${ }^{7}$ Toutes les occurrences de AUCUN ont été extraites, analysées et classées. La table présentée cidessous donne un aperçu de la distribution des divers emplois de AUCUN dans le corpus journalistique.

Table 1

\begin{tabular}{|l|l|l|l|l|l|}
\hline [aucun N] & $\begin{array}{l}\text { Enoncés } \\
\text { existentiels }\end{array}$ & $\%$ & $\begin{array}{l}\text { Enoncés } \\
\text { déclaratifs }\end{array}$ & $\%$ & Total \\
\hline sujet & 104 & 44 & 134 & 56 & 238 \\
\hline sujet réel & 43 & 100 & 0 & 0 & 43 \\
\hline objet & 193 & 89 & 24 & 11 & 217 \\
\hline \hline Total & 340 & 68 & 158 & 32 & $498^{8}$ \\
\hline
\end{tabular}

On le constate, sur les quelques 500 occurrences relevées, environ deux tiers se trouvent dans un énoncé existentiel et un tiers dans un énoncé déclaratif. Notons également que si la distribution des deux types de AUCUN est plus ou moins égale en fonction de sujet (44 pour cent versus 56), l'interprétation non référentielle l'emporte largement en fonction d'objet direct (89 pour cent versus 11). ${ }^{9}$ Dans la construction impersonnelle, AUCUN N ne reçoit nulle part une interprétation référentielle, ce qui était prévisible. Cette répartition m'a amenée à concentrer mon analyse sur les SN sujets, où les deux types de AUCUN entrent véritablement en concurrence l'un avec l'autre. Ceci ne m'empêchera pas, cependant, d'aborder de temps en temps des problèmes posés par les SN objets.

Pour une analyse des conditions réglementant la référentialité des SN sujets déterminés par AUCUN, on peut envisager au moins deux options qui seront commentées à tour de rôle dans ce qui suit. La première consiste en une approche pragmatique, la seconde consiste à élucider certains rapports sémantiques au sein des énoncés véhiculant une occurrence de AUCUN. 


\section{L'approche pragmatique}

être en mesure de prendre une décision quant au domaine de définition de AUCUN : le quantificateur, opère-t-il ou n'opère-t-il pas sur un sous-ensemble fini d'objets spécifiques (de la catégorie dénotée par N), présupposé par le locuteur? Cette décision peut (mais ne doit pas, comme on le verra tout à l'heure) se baser sur diverses considérations d'ordre discursif. Le cas le plus évident fait intervenir une mention explicite de ce sous-ensemble, fait illustré par les énoncés (10)-(13). Les SN désignant les ensembles en question ont été mis en italiques.

10 AUCUN de ces trois points ne mènera à des difficultés insurmontables. (Le Soir, 880412)

11 Je trouvais cinq habits étalés sur mon lit en rentrant pour me changer. Mais AUCUN ne m'allait vraiment. (JOL, 008-05)

12 Decaux passe en revue les diverses hypothèses avancées à ce jour, dont AUCUNE n'est pleinement satisfaisante. (Le Soir, 880408)

13 Mais AUCUNE SCENE ne plaisait autant à Vendredi que celle du début, quand il fuyait les Araucans qui voulaient le sacrifier [...]. (MT, 213)

Avec la construction AUCUN DES N, dans (10), la mention de ce sous-ensemble s'incorpore au SN quantifié lui-même. Celle-ci peut également apparaitre dans un énoncé (immédiatement) précédent, où elle servira d'antécédent à AUCUN, soit directement, comme dans (11), soit par l'intermédiaire de l'anaphorique DONT, comme dans (12). Dans un énoncé comportant une comparaison, elle surgit derrière que, comme le montre (13).

Or, le sous-ensemble présupposé n'est pas nécessairement mentionné en toutes lettres. Son existence peut s'établir par voie inférentielle. L'exemple (14) illustre cette situation un peu plus complexe et pourtant tout à fait banale dans la pratique langagière.

14 Chez Néca, AUCUNE TABLE n'était libre à la terrasse [...]. (PMO, 101-02)

Pour un lecteur du roman d'où est tiré cet exemple ${ }^{11}$, une lecture référentielle de aucune table s'impose. Cette lecture est assurée par un raisonnement reposant sur les prémisses suivantes : $1^{\circ}$ Néca est un personnage de l'univers fictif imaginé par le romancier (c'est un référent dont l'existence a été établie dans le contexte précédent). $2^{\circ}$ Néca est le propriétaire d'un café (information due au contexte précédent). $3^{\circ}$ Dans un café, il y a des tables et des chaises à l'usage des consommateurs et celles-ci peuvent se trouver sur la terrasse (ceci fait partie de nos connaissances stéréotypées sur le monde, notre savoir « encyclopé-dique »). Ces prémisses garantissent la conclusion qu'il y a effectivement des tables chez Néca. Par conséquent, il est question d'un ensemble fini et spécifique de tables, ce qui fait de (14) un énoncé déclaratif.

De cette description, si rudimentaire soit-elle, il ressort que, pour interpréter un énoncé donné contenant une occurrence de AUCUN, il ne suffit pas toujours d'avoir accès à ce seul énoncé. Il faut encore faire appel à un arrière-plan d'informations obtenues ou inférées du contexte discursif, ainsi qu'à des connaissances diverses de caractère linguistique ou encyclopédique. De nombreux chercheurs sont engagés dans une description pragmatique de problèmes qui rejoignent celui-ci. Ne citons que les travaux d'Anne Reboul - inspirés de la théorie de la pertinence élaborée par Sperber et Wilson (1986) - qui traitent de l'attribution de référents aux anaphores pronominales, en vue d'un traitement automatique. ${ }^{12}$ 

sommes en présence d'un problème qui relève dans une certaine mesure de la pragmatique. Or, il suffit d'examiner isolément les énoncés fournis par les corpus pour constater qu'il n'est pas toujours nécessaire de se reporter au contexte discursif ou situationnel pour interpréter correctement un énoncé donné. Je dirais plutôt que, bien souvent, on peut s'en passer tout à fait. En témoigne par exemple l'énoncé (14) analysé tout à l'heure. En effet, je doute fort que, même en l'absence de toute considération pragmatique préalable, on puisse lui attribuer une interprétation existentielle. Faut-il en conclure qu'à côté des facteurs pragmatiques déjà évoqués, des facteurs sémantiques entrent également en jeu dans l'interprétation des énoncés munis d'un SN en AUCUN, peut-être de façon redondante ? C'est pour trouver un début de réponse à cette question que j'ai choisi de me concentrer dans cet article sur quelques aspects sémantiques des énoncés en AUCUN. Plus précisément, il s'agira de propriétés sémantiques chez certaines unités lexicales, surtout en proposition existentielle.

Signalons tout de suite qu'une analyse sémantique des items lexicaux apparaissant conjointement avec AUCUN est un problème délicat dans la mesure où elle nécessite la prise en compte d'un grand nombre de faits et d'hypothèses. Aussi la description proposée ici ne prétend-elle nullement à l'exhaustivité. A la vérité, elle ne constitue qu'un commen-cement de réflexion, destiné surtout à inciter à un dialogue scientifique.

\section{Facteurs sémantiques}

\section{AUCUN N en proposition existentielle}

\section{Les verbes dits existentiels}

Quels types de facteurs non pragmatiques jouent donc un rôle dans le processus d'attribution de référents aux SN en AUCUN? Une hypothèse qui se présente naturellement si l'on veut faire entrer en ligne de compte la sémantique lexicale, c'est qu'il existe une ou des classes de verbes susceptibles d'imposer une lecture donnée du SN sujet introduit par AUCUN. (On laissera de côté ici les structures impersonnelles, dans la mesure où leur interprétation ne pose pas de problème à cet égard.) En ne tenant compte pour le moment que des verbes intransitifs des corpus figurant en énoncé existentiel, on voit apparaître la classe des verbes dits existentiels comme un candidat plausible. Arrêtons-nous un instant sur le verbe exister qui est le membre prototypique de cette classe. Les données fournies par les deux corpus semblent indiquer que ce verbe se combine invariablement avec un sujet non référentiel, quelle que soit la nature du nom tête. L'interprétation référentielle serait-elle exclue avec exister? En tout cas, on constate qu'en interprétant référentiellement le SN sujet, on provoque une contradiction. Cette lecture est incompatible avec le sens du verbe exister englobé dans le champ de la négation. Considérons à titre d'exemple (15), où (a) fait apparaître un sujet concret et (b) un sujet abstrait :

15 a. [...] aucun box assez vaste n'existe dans aucun tribunal pour accueillir tous les coupables de cette débâcle. (Le Soir, 880407)

b. [...] aucun accord d'extradition n'existe entre le Honduras et les Etats-Unis. (Le Soir, 880409)

$$
\text { Muni d'un SN sujet abstrait comme par exemple aucun accord d'extradition, quelle fonction }
$$
ce verbe pourrait-il avoir, sinon d'asserter la non-existence d'accords d'extradition dans 
la situation évoquée par (15b) ? En fait, sélectionner l'interprétation référentielle du SN sujet équivaut à présupposer d'abord l'existence d'un ou de plusieurs accords d'extradition entre les pays cités, pour affirmer ensuite que, parmi ces accords, il n'y en a aucun qui existe, ce qui est absurde. La même contradiction se retrouve dans (15a), dont le sujet a un référent concret, dès qu'on s'avise d'imposer une lecture référentielle du SN introduit par AUCUN.

Comment délimiter la classe des verbes existentiels? En qualifiant exister de prototypique, j'ai voulu souligner le fait que parmi tous les membres de cette classe, c'est ce verbe-là qui comporte le contenu sémantique le plus réduit : il désigne l'existence tout court. Quant aux autres membres, ils semblent désigner l'existence et quelque chose en plus. En quoi consiste donc ce «plus»?

En ce qui concerne rester et subsister, par exemple, ces verbes désignent une existence qui se poursuit (sous la négation: une existence qui a cessé), ce qui ne change en rien l'impossibilité de la lecture référentielle. Ainsi on se contredit toujours en affirmant à propos des obstacles dont il est question dans (16) que, tout en existant, ils ne subsistent plus :

16 Après la rencontre Gorbatchev-Najibullah, plus aucun obstacle ne subsiste à la signature d'un accord final entre le Pakistan et l'Afghanistan. (Le Soir, 880408)

Avec entrer, dans (17), l'accent est mis sur une présence de caractère plus ou moins permanent. Sous l'effet de la négation cela revient à asserter une absence. Encore une fois, la lecture référentielle se révèle exclue.

17 a. Il s'est révélé qu'aucune substance active n'entrait dans leur composition. (La Tribune de Genève, 880303)

b. [...] le vieux fut entouré, interrogé avec une curiosité sérieuse ou goguenarde, mais où n'entrait aucune indignation. (Le Soir, 880413)

31 Il peut également s'agir d'une existence ou d'une présence pour ainsi dire inchoative, en ce cens qu'elle fait son " entrée en scène ». Etant donné le contexte négatif, le résultat est de nouveau une assertion d'absence interdisant toute présupposition quant à l'existence du référent du sujet.

18 a. Aucun nom européen n'apparaît dans les 30 premiers. (La Tribune de Genève, 880517)

b. Aucun élément neuf n'est apparu dans ce dossier depuis quatre ans. (Libre Belgique, 860212)

c. Pour autant bien sûr que ne survienne aucun imprévu. (Libre Belgique, 860214)

d. Mais les soirées devenaient pénibles à passer ensemble, bien qu'aucun désaccord grave n'eût surgi. (Le Soir, 881413)

e. Il ouvrit la bouche, mais aucun son ne sortit. (CAU, 053-13)

Dans le cas des exemples réunis sous (19), il s'agit d'un mode de présence particulier, caractéristique des référents appartenant à la catégorie dénotée par le nom du SN sujet. Cette présence est transformée en absence par la négation.

19 a. Aucun souffle d'air ne passait dans la brume épaisse endormie sur le fleuve. (Le

Soir, 880413) (cf. 2a ci-dessus)

b. Le rivage était complètement désert; sur la route, aucune voiture ne passait, à cause des vagues qui se répandirent sur la chaussée. (JCL, -168-29)

c. [...] aucune information fiable sur la situation réelle du pays n'aurait pu circuler. ( La Tribune de Genève, 8803X1)

d. Effectivement, tout autour des deux petits bâtiments, c'est la désolation ; aucun brin d'herbe ne pousse et des barbelés isolent de plus en plus les bonnes sœurs. ( Libre Belgique, 871127) 
d'une part que les énoncés discutés jusqu'ici, en refusant la lecture référentielle sont tous univoques du point de vue de l'interprétation du SN sujet; d'autre part qu'ils admettent tous une paraphrase impersonnelle. Dans une étude consacrée à la phrase impersonnelle, Kesik (1986, p. 258) fait observer au sujet des verbes acceptant d'entrer dans cette construction qu'ils ont "une charge sémantique très faible » et qu'ils désignent l'existence, la présence ou l'absence, «avec des nuances aspectuelles éventuelles ». Ces propriétés semblent coïncider avec celles que nous avons discernées chez les verbes apparaissant dans les énoncés existentiels pourvus d'un SN sujet en AUCUN. Or, le fait d'admettre une paraphrase impersonnelle n'est pas un critère décisif. Comme on le sait, un énoncé qui fait figurer la copule et un syntagme adjectival ou prépositionnel en fonction d'attribut n'autorise jamais la paraphrase impersonnelle. ${ }^{13} \mathrm{Et}$ pourtant ces énoncés-là n'excluent nullement l'emploi d'un SN en AUCUN non référentiel, fait illustré par (20a-c) :

20 a. Aucune réforme économique ne sera réalisable et soutenue en Pologne sans

l'introduction du pluralisme syndical. (Libre Belgique, 871126)

b. Après, plus aucun remboursement ne sera possible [...]. (Le Soir, 880405)

c. [...] aucune baisse des taux hypothécaires n'est en vue. (La Tribune de Genève, 880302I)

semble donc que la lecture non référentielle s'explique davantage par le sens du prédicat, qui désigne une existence potentielle, pas encore réalisée. Une nuance sémantique apparentée se retrouve par ailleurs avec le verbe prévoir :

21 Celle-ci avait demandé au promoteur des garanties concernant les parkings.

Aucun emplacement n'est prévu [...]. (Le Soir, 880406)

facteur responsable de l'incapacité de ces prédicats à faire naître une lecture référentielle est vraisemblablement le caractère extrêmement réduit de leur contenu sémantique. ${ }^{14}$ Plus un verbe manque de plénitude sémantique, plus cela manque de sens de présupposer l'existence du SN sujet. En effet, dans une perspective de pertinence communicative, des énoncés comme ceux qu'on vient de voir se justifient mal, sinon en tant qu'assertions de non existence. Envisagés comme des énoncés déclaratifs, ils commencent par attirer l'attention sur un référent particulier qu'ils abandonnent ensuite sans apporter d'information à son sujet, ou peu s'en faut.

A la limite, ces verbes remplissent surtout une fonction syntaxique comme porteurs de marques de temps et de personne. Par conséquent, on peut facilement les supprimer, sans altérer le contenu informationnel global. Ce qui est modifié, c'est plutôt le style, dont la brévité résultante rappelle les titres de journaux ou le débit un peu coupé de certains écrivains. Considérons quelques exemples à titre d'illustration :

22 Aucun accord d'extradition entre le Honduras et les Etats-Unis. (cf. 15b)

Aucun nom européen dans les 30 premiers. (cf. 18a)

23 Il ouvrit la bouche, aucun son. (cf. 18e)

Le rivage était complètement désert ; sur la route, aucune voiture. (cf. 19b)

37

En examinant les énoncés incomplets fournis par les corpus - c'est-à-dire ceux dépourvus de verbe (cf. note 8) - on constate qu'en aucun cas ils n'autorisent une lecture déclarative. En voici deux exemples :

24 Rassurons immédiatement parents et éducateurs : aucune arrière-pensée immorale ici. (Le Soir, 880412)

25 Dans tout cela, aucun conflit de compétences avec les organismes publics et diplomatiques. (Le Soir, 880411) 

Des hypothèses relatives au nom tête se laissent difficilement examiner à partir des verbes exemplifiés dans la section précédente, dans la mesure où ceux-ci s'accompagnent invariablement d'un sujet dont le référent n'existe pas, du moins dans les corpus étudiés. En se tournant vers les énoncés transitifs, on constate cependant que la distribution des noms révèle un déséquilibre intéressant. La proportion des noms tête marqués du trait (concret) est très élevée en proposition existentielle et faible en proposition déclarative, où dominent les noms marqués (+ animé). Les exemples suivants sont typiques, (31) parce 
qu'il constitue un énoncé existentiel muni d'un sujet (- concret), (32) parce qu'il est déclaratif et muni d'un sujet (+ animé) :

31 Aucun entretien régulier n'a été effectué et maintenant des rénovations coûteuses doivent être réalisées. (La Tribune de Genève, 8803X1)

32 Aucun berger de son voisinage n'avait accepté de prendre en charge son troupeau pendant la durée de son service militaire. (Le Soir, 880411)

L'énoncé (33), par contre, est peu typique dans la mesure où il comporte un SN sujet (concret) tout en restant déclaratif :

33 A leur grand désespoir, aucun traitement ne réussit. (Le Soir, 880412)

Les SN non référentiels pourvus d'un nom tête (+ animé), comme dans (34), sont tout à fait exceptionnels. Et il y en a relativement peu qui, à l'instar de écrit dans (35), portent le trait (+ concret) :

34 Aucune victime n'a été signalée à bord d'un autre navire atteint [...]. (La Tribune de Genève, 880516)

35 Bien qu'aucun écrit n'ait été retrouvé dans l'habitation du couple, il semble que ce drame ait été décidé de commun accord. (Libre Belgique, 860212)

Ces observations sont certes intéressantes. Etant donné l'absence d'une distribution complémentaire parfaite, on ne peut cependant prédire avec sûreté l'interprétation d'un $\mathrm{SN}$ donné sur la base des traits sémantiques discutés. Une solution un peu différente consiste à avancer une hypothèse faisant intervenir le rapport substantif/verbe. Pour les $\mathrm{SN}$ sujets introduits par AUCUN, il s'agit plus précisément du rapport N-V représenté par le schéma (36a) et, pour les SN objets correspondants, du rapport V-N représenté par (36b). (Si SN2 a été mis entre parenthèses dans (a), c'est pour faire rentrer dans ce schéma les nombreux cas où AUCUN $\mathrm{N}$ fonctionne comme sujet d'un verbe passif.)

36 a. [SN1 aucun $\mathrm{N}]$ ne $\mathrm{V}(\mathrm{SN} 2)$

b. SN1 ne $\mathrm{V}$ [SN2 aucun $\mathrm{N}]$

Considérons maintenant le suivant choix d'énoncés existentiels, qui font tous figurer un SN en AUCUN pourvu d'un nom tête marqué (- concret). Ceux de (37) s'alignent sur le schéma (36a) et ceux de (38) sur le schéma (36b).

37 J'ai toujours dit qu'aucune étude sérieuse n'avait été faite et que j'avais été trompé. (Le Soir, 880402)

Mais aucune décision concrète n'a encore été prise. (Libre Belgique, 860213)

Nous avons bien un agent à Gênes, mais aucun contact n'a été pris [...]. (La Tribune de Genève, 880517)

Aucune mesure particulière n'a été prise. (Le Soir, 880411)

Aucun détail n'a été donné sur la manière dont il a pu être pris [...]. (La Tribune de Genève, 880229)

38 En tout cas, je n'ai aucune crainte sur l'issue de notre opération. (Le Soir, 880414) [...] les prisonniers capturés [...] n'ont aucun espoir de regagner leur foyer. (Le Soir , 880407)

L'eau était blanche de remous, les vagues n'avaient plus aucun sens dans cet espèce d'énorme chaudron du diable. (Libre Belgique, 860216)

Bref, notre interlocuteur ne fait aucune confiance au groupe de travail. (La Tribune de Genève, 880517)

Les médecins n'ont encore fait aucune déclaration sur l'état de santé des bébés. (Le Soir, 880406)

Nous n'avons fait nous-mêmes aucune proposition concrète [...]. (La Tribune de Genève, 880517)

Elle ne prend aucun risque, et c'est peut-être ce que l'on peut regretter. (Le Soir, 880413)

[...] les recherches de la police n'ont donné aucun résultat. (Libre Belgique, 860212) 
les exemples cités ont ceci en commun de faire apparaitre ce que l'on appelle habituellement un verbe "support». Par verbe support j'entends ici un verbe sémantiquement peu autonome comme avoir, faire, donner ou prendre, qui se combine avec un complément d'objet direct ${ }^{15}$ pour former une unité sémantique souvent lexicalisée par un verbe seul, comme importer pour avoir de l'importance, gifler pour donner une gifle, commenter pour faire un commentaire, finir pour prendre fin, etc.

5

Une lecture déclarative des énoncés cités est peu concevable en raison du peu de contenu informationnel ${ }^{16}$ véhiculé par ces verbes, qui, au niveau sémantique, réalisent une espèce de fusion avec le SN qu'ils régissent. ${ }^{17} \mathrm{Je}$ proposerai donc l'hypothèse suivante: en combinant un substantif (- concret) - incorporé dans un SN déterminé par AUCUN - avec un verbe support, on obtient une phrase existentielle.

donné cette hypothèse, que dira-t-on des énoncés suivants où reviennent les mêmes noms que dans (37-38), quoique combinés avec un verbe différent? Tout en n'autorisant qu'une lecture existentielle, ces énoncés font figurer un SN sujet ou objet muni d'un nom tête abstrait, mais aucun des verbes support classiques cités ci-dessus? Intuitivement, on dirait que les verbes utilisés assument une fonction analogue à celle assumée par les verbes de (37) et de (38). Le problème qui se pose, manifestement, c'est la délimitation de la classe des verbes support.

39 Aucune étude approfondie et fiable n'a été menée pour permettre au citoyen de se déterminer à bon escient. (La Tribune de Genève, 870626)

[...] aucune décision du comité n'était attendue. (Le Soir, 880411)

Aucune mesure n'a été mise en œuvre en temps utile [...]. (Le Soir, 880413)

Aucun détail n'a été fourni sur la nature des actes reprochés au suspect. (Le Soir, 880406)

40 Je n'éprouve aucune crainte au sujet des époux Kihan [...]. (RPI, 231-08)

[...] le chapitre sur l'éducation ne laissait aucun espoir de sortir de cet enfer monosexuel. (JDL, 005-06)

La Terre bouge, elle ne m'inspire aucune confiance [...]. (NOB, 651-79)

On n'obtint aucun résultat. (Le Soir, 880413)

En examinant de plus près les données fournies par les corpus, on découvre que la plupart des substantifs marqués (- concret) semblent posséder leur propre verbe support, qui n'est pas nécessairement avoir, faire, donner, poser, prendre, etc. Avec (41), semble-t-il, on se trouve devant des exemples dont les verbes n'appartiennent guère à la catégorie des verbes support classiques.

41 Mais les grands magasins disent qu'aucune rançon n'a été exigée. (Le Soir, 880408)

Aucun terme n'avait été fixé à l'application de ces facilités. (Le Soir, 880412)

Quant à la participation du Maccabi Tel-Aviv, qui s'assimilait furieusement à un

cadeau empoisonné, elle n'a causé aucun dégât. (Le Soir, 880408)

Pascal Legac, 23 ans, n'a opposé aucune résistance. (La Tribune de Genève, 880303)

Si j'insiste néanmoins pour les déclarer verbes de support, c'est à cause de la relation très étroite qu'ils entretiennent avec le nom tête du SN déterminé par AUCUN. En témoigne le fait que, dans le Petit Robert, ces verbes entrent dans la définition même du substantif auquel ils se rapportent dans (41), du moins dans la définition d'une de ses acceptions. ${ }^{18}$ Ainsi le verbe exiger se retrouve dans le «definiens » de rançon, alors que les verbes fixer, causer et opposer s'utilisent dans ceux de terme, dégât et résistance, respectivement. Sous le coup de cette dépendance, le verbe semble se vider de son contenu sémantique propre. 
si l'on veut qu'elle soit vraiment utile à l'analyse proposée. Or, notons qu'une tentative de dresser la liste de l'ensemble des verbes support est vouée à l'échec. Une telle liste serait très longue et composée d'éléments trop hétéroclites pour permettre des générali-sations intéressantes. Considérons quelques exemples pour illustrer cette objection. On remarquera au passage que les verbes ont tendance à se regrouper d'après le sémantisme $\mathrm{du}$ nom tête avec lequel ils entrent en relation. Ainsi on trouvera divers verbes synonymes de faire avec les noms dénotant une activité, comme dans (42). Et lorsque le nom dénote une entité susceptible de se présenter sous forme linguistique, comme dans (43), l'on voit surgir des verbes comme préciser, formuler, communiquer, etc.

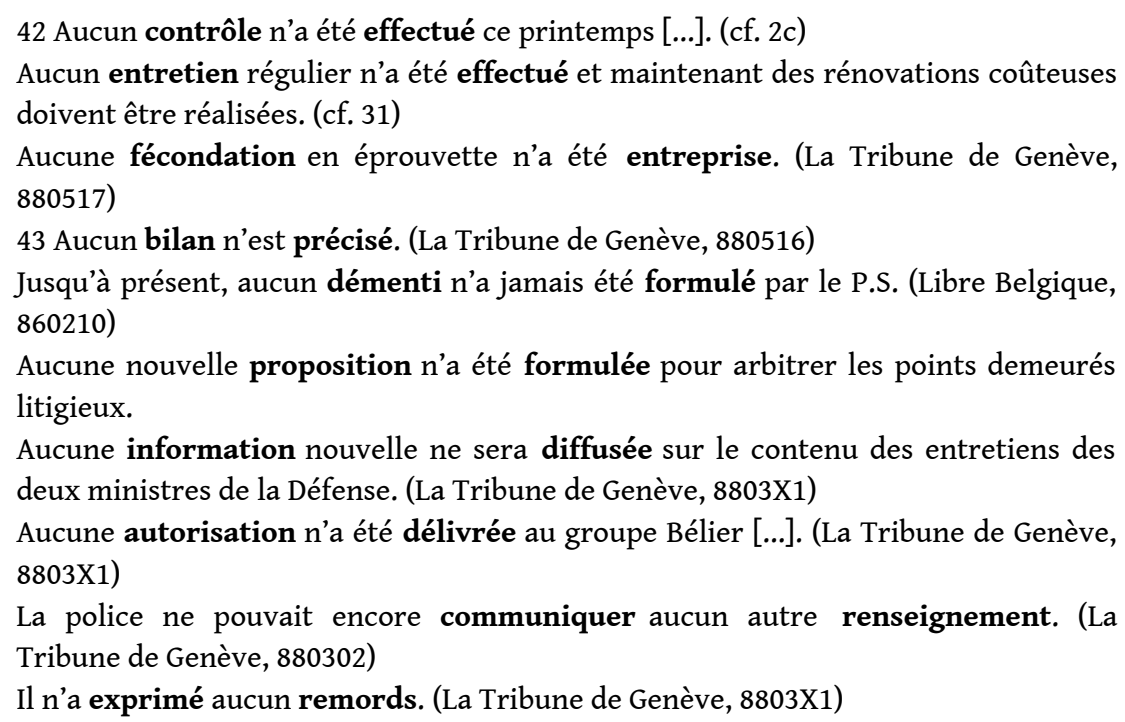

D'autres verbes seront sélectionnés avec un nom désignant une somme d'argent (cf. 44), un traitement pénible (cf. 45), un sentiment (cf. 46) etc.

44 Aucun appointement n'est versé aux membres de l'association [...]. (Le Soir, 880407)

[...] aucun budget n'est prévu pour ces travaux. (Le Soir, 880407)

[...] il ne pourra demander ni accepter aucune rétribution des abonnés. (Libre Belgique, 871127)

45 Aucun sévice physique ou sexuel n'aurait été subi par la gamine. (Le Soir, 880408)

Les malfaiteurs sont repartis avec 12.000 francs en poche, sans avoir prodigué aucune violence envers le personnel. (La Tribune de Genève, 880518)

46 [...] je n'éprouve aucun sentiment d'agressivité à l'égard de mon père. (RPI, 163-02)

Bref, le régime castriste ne montre aucune sympathie pour la glasnost de Gorbatchev. (La Tribune de Genève, 880307)

On pourrait continuer indéfiniment cette énumération: plus on cite de substantifs différents, plus la liste des verbes support s'allonge. De cette démonstration, il ressort cependant que cela n'a pas beaucoup de sens d'adopter une démarche interprétative consistant à associer un $\mathrm{SN}$ donné du type AUCUN $\mathrm{N}$ à un verbe prélevé sur un ensemble hétéroclite de verbes dits de support. En fait, on risque de trouver sur cette liste bon nombre de verbes susceptibles d'apparaître en énoncé déclaratif. 
59 Etant donné que la sélection du verbe dépend crucialement du substantif choisi, une méthode plus efficace consisterait à prévoir une analyse qui ait accès à un lexique renseignant systématiquement sur les verbes support avec lesquels se combine chaque substantif. Ce serait par exemple un lexique du type proposé par Mel'cuk et ses collaborateurs, où le rapport V-N peut être défini en termes de "fonctions lexicales ». ${ }^{19}$ Citons à ce propos Leonard Babby (1980, p. 139):

We can now define lexical functions in more general terms. There are essentially two sets of words in the lexicon of any natural language: autonomous words and words which serve as the lexical correlate of other words. The relationship between an autonomous word and its lexical correlate is a dependency which has been called a lexical function. The autonomous word is the argument of this function, and the corresponding correlate is its value.

60 Soient, par exemple, un substantif marqué (- concret) et une fonction lexicale, OPER, qui prend pour arguent un $\mathrm{N}$ (- concret) et rend comme valeur un verbe transitif susceptible de fonctionner comme verbe support. Appliquée au substantif but, dans son acception sportive, OPER donnera la valeur marquer, appliquée à mesure, elle donnera prendre ou mettre en œuvre, et ainsi de suite. ${ }^{20}$

61 Compte tenu de ces observations sur les verbes support, je proposerai la reformulation suivante de l'hypothèse avancée tout à l'heure: étant donné un substantif marqué (concret) qui s'incorpore à un SN introduit par le quantificateur négatif AUCUN, une interprétation non référentielle de ce SN s'impose lorsqu'il est combiné avec un de ses verbes support (spécifiés par le lexique).

L'exemple (34), repris ici pour plus de commodité, me semble exiger une dernière modification de cette hypothèse.

34 Aucune victime n'a été signalée à bord d'un autre navire atteint, le britannique

Burmah Endeavour. (La Tribune de Genève, 880516)

63 Avec (34), on se trouve devant un énoncé existentiel pourvu d'un SN sujet sous-catégorisé (+ animé), situation qui n'est pas prévue par l'hypothèse proposée. Or, signaler dans (34) semble fonctionner comme verbe support par rapport à victime. Il serait possible, me semble-t-il, d'inclure ce cas et des cas analogues en modifiant légèrement notre hypothèse. On atteindra ce but en faisant intervenir non pas simplement des substantifs abstraits, mais plutôt des substantifs syncatégoréma-tiques. J'ai emprunté le terme syncatégorématique à Georges Kleiber, qui établit une distinction entre noms syncatégorématiques et noms catégo-rématiques et disant que :

[...] a) sont catégorématiques les substantifs qui peuvent repérer par eux-mêmes une catégorie référentielle d'occurrences individuelles stable ou homogène, et b) sont syncatégorématiques les substantifs qui ne peuvent pas, par eux-mêmes, c'est-à-dire sans l'aide d'autres repères, repérer les référents particuliers auxquels ils peuvent s'ap-pliquer. En résumé, la référence particulière d'un substantif catégorématique peut être repérée grâce au nom lui-même, alors que la référence particulière d'un substantif syncatégorématique requiert des repères antérieurs ou, pour parler comme Strawson, une méthode ou un principe de groupement antécédents. (Kleiber, 1981, p. 40)

64 En vertu de la définition citée, il me semble que victime doive se classer parmi les noms syncatégorématiques. En fait, la définition que donne Kleiber n'exclut aucunement les noms désignant des entités concrètes ou animées (il le souligne lui-même ${ }^{21}$ ), la propriété cruciale étant celle de renvoyer à une catégorie référentielle hétérogène. 


\section{AUCUN $\mathrm{N}$ en proposition déclarative} souvent - mais pas toujours - avec des verbes dont la structure sémantique est plus riche et plus complexe que celle des verbes existentiels ou de support. Le verbe utilisé impose habituel-lement l'emploi d'un SN sujet (ou objet) sous-catégorisé (+ concret) ou (+ animé). Notons aussi que certains verbes réfractaires à une interprétation existentielle (comme résister - cf. $4 \mathrm{a}$ - ou réussir) entraînent la lecture déclarative, même combinés avec un $\mathrm{SN}$ sujet syncatégorématique comme aucune victime. Que l'on compare (47) et (48) à (34) cité ci-dessus :

47 Aucune victime de l'incendie n'avait résisté à la tentation de sauter par la fenêtre.

48 Aucune victime n'a réussi à enfiler la veste de sauvetage.

Que se passe-t-il quand un SN muni d'un substantif catégorématique se combine avec un verbe support? En principe, ces substantifs n'ont pas de verbes support, la raison en étant, bien entendu, que leurs référents peuvent entrer dans un nombre illimité de relations très diverses. Ceci dit, ils apparaissent tout de même fréquemment avec des verbes qui, par ailleurs, fonctionnent comme verbe support avec un nom syncatégorématique. On observera que, dans ce cas-là, le résultat est souvent un énoncé ambigu. En fait, comment faut-il interpréter l'énoncé (49), dans lequel il est question de la pyramide de Sakkara?

49 Le miracle, c'est que l'on connaît son architecte : Imhotep. Combien de temps mit-il à la construire? Aucune annale ne nous le précise. (Le Soir, 880408)

Est-ce que cet énoncé fait entendre qu'il existe des annales de l'époque d'Imhotep, mais que ces annales ne nous renseignent pas sur le temps qu'on mit à construire la pyramide? Ou est-ce qu'il veut dire qu'il n'y a pas d'annales pour nous le préciser? Pour trancher cette question, il faut faire appel à nos connaissances sur l'histoire de l'ancienne Egypte, à moins que le contexte discursif ne nous en informe. Dans les deux cas, c'est la pragmatique qui intervient pour régler le problème. Considérons un dernier exemple, signifiant que si les routiers passent sans s'arrêter à l'Auberge du Régal, c'est qu'il n'existe aucun feu rouge pour les faire arrêter à cet endroit :

50 Insolite en cet endroit, l'Auberge du Régal regarde passer les routiers que n' arrête aucun feu rouge. (CET, 271-21 227)

Or, (50) accepte également une lecture signifiant que cette auberge regarde passer des camionneurs ayant la fâcheuse habitude de brûler tout feu rouge qui se dresse sur leur chemin. Selon la première interprétation, il n'y a donc pas de feux rouges dans le coin. La 
seconde reste indécise à ce sujet. En tout cas : qu'il y en ait ou qu'il n'y en ait pas, ce n'est pas la présence d'un feu rouge qui ferait en sorte que les routiers s'arrêtent.

Une solution sémantique du problème interprétatif posé par (49) et (50) me semble difficilement concevable. Pour lever l'ambiguïté décrite, il convient sans doute d'avoir recours à une analyse pragmatique qui fasse intervenir ou bien le contexte discursif ou bien diverses connaissances du monde de nature encyclopédique.

\section{En guise de conclusion}

72 Cet exposé part de la constatation que les SN déterminés par le quantificateur AUCUN s'ouvrent, en principe, à deux lectures distinctes. La première, qui est non référentielle, correspond à l'analyse traditionnelle des SN en AUCUN. Elle se rattache aux énoncés existentiels, c'est-à-dire aux énoncés exprimant une assertion de non existence. La seconde est référentielle, dans la mesure où elle fait intervenir un ensemble fini d'éléments spécifiques dont l'existence est présupposée par le locuteur. Les SN référentiels se retrouvent dans les énoncés déclaratifs, où l'assertion porte plutôt sur l'action, le processus ou l'état désignés par le prédicat. Etant donné l'ambiguïté potentielle des SN introduits par AUCUN, le problème se pose d'identifier les facteurs responsables de l'une et de l'autre interprétation. Comme nous l'avons observé, tout traitement automatique du français nécessite une description de ce phénomène.

Pour ce genre de problème, le recours à une solution en termes pragmatiques peut sembler aller de soi. Sans écarter cette possibilité, j'ai cependant essayé ici d'explorer les facteurs sémantiques intraphrastiques susceptibles d'avoir un impact sur l'interprétation des SN en AUCUN. Voici, en résumé les conclusions d'une analyse basée sur toutes les occurrences de AUCUN trouvées dans deux corpus informatisés.

Une interprétation non référentielle s'impose lorsque chacune des conditions suivantes est remplie : a) le nom tête du SN déterminé par AUCUN est à la fois marqué du trait (concret) et syncatégorématique; b) le SN déterminé par AUCUN est le complément d'objet d'un verbe transitif, éventuellement mis au passif ; c) le verbe accompagnant le SN en AUCUN est sélectionné parmi les verbes support qu'un lexique exhaustif spécifie pour le nom tête du $\mathrm{SN}$ en question.

Sujet d'un verbe intransitif appartenant à la catégorie des verbes dits existentiels, un SN en AUCUN a une forte tendance à recevoir une interprétation non référentielle. Les conditions précises de la non apparition de cette lecture restent à élucider.

En revanche, la lecture référentielle s'impose lorsque a) la structure sémantique du verbe manifeste assez de richesse pour assurer l'autonomie de celui-ci; b) le nom tête du SN déterminé par AUCUN est catégorématique et marqué des traits (+ animé) ou (+ concret) ; c) le verbe exige un SN sujet (ou objet) ainsi sous-catégorisé. Il reste à examiner si la condition a) est à la fois nécessaire et suffisante.

La présence d'un verbe qui fait subir peu de restrictions sémantiques - ou aucune - à un SN en AUCUN dépendant de lui a tendance à produire une ambiguïté qui sera levée la plupart du temps par des renseignements fournis par le contexte discursif ou situationnel, qu'il s'agisse d'un SN sujet comportant un nom tête syncatégorématique ou catégorématique. 


\section{BIBLIOGRAPHIE}

Babby, L., 1980, Existential Sentences and Negation in Russian, Ann Arbor, Karoma Publishers.

Dahl, Ö., 1973, « On the Semantics of Quantified Noun Phrases and Related Problems », Göteborg, Département de linguistique générale, Université de Göteborg.

Heldner, C., 1989, « Le sujet grammatical dans la construction impersonnelle », Studia Neophilologica, 61, pp. 89-115.

Heldner, C., 1992, « Sur la quantification négative », Langue française, 1992, 94, pp. 80-92.

Kesik, M., 1986, « Description de la phrase impersonnelle entre la syntaxe, la sémantique et la rhétorique » in Actes du XVIIe congrès international de linguistique et philologie romanes, pp. 251-263, Publications de l'Université de Provence.

Kleiber, G., 1981, Problèmes de référence : Descriptions définies et noms propres. Recherches linguistiques. Etudes publiées par le Centre d'Analyse Syntaxique de l'Université de Metz. Paris, Klincksieck.

Mel'cuk, I.A. \& A.K. Zolkovskij, 1970, « Toward a Functioning 'Meaning-Text' Model of Language ", Linguistics, 57, pp. 10-47.

Picabia, L., 1987, « Quand y a-t-il générique ? » in Rencontre(s) avec la généricité. Recherches linguistiques XII, G. Kleiber (éd.), pp. 235-258, Paris, Klincksieck.

Reboul, A., 1988/1989, « Pragmatique de l'anaphore pronominale », in SIGMA, 12-13, pp. 197-231, Publications de l'Université de Provence.

Reboul, A., 1989, « Résolution automatique de l'anaphore pronominale » in Modèles de discours, pp. 173-192, Berne-Francfort-Paris, Peter Lang.

Sperber, D., \& Wilson, D., 1986, Relevance. Communication and Cognition, Oxford, Basil Blackwell.

\section{NOTES}

1. Plus exactement, je soutiens que l'emploi de PAS $D E+N$ se restreint aux énoncés exprimant une proposition existentielle, tandis que AUCUN $+\mathrm{N}$ apparait aussi bien en proposition existentielle qu'en proposition déclarative.

2. Cf. par exemple Picabia (1987, p. 240): « [...] une construction existentielle standard comme il existe a pour fonction d'asserter ou de nier l'existence d'objets »; ou Babby (1980, p. 63): «The crucial difference between these declarative and existential sentence pairs can be summarized as follows: an AES [affirmative negative sentence] asserts that the subject of the sentence exists (the temporal and/or locative adverbial expressions determine whether the existential assertion is « absolute » or « relative »); « [...] the primary function of an NES [negative existential sentence] is to assert that its subject does not exist, i.e., that the subject NP does not have an identifiable referent ».

3. Comme le note Kleiber (1981), des énoncés comme Il existe des chevaux ou Il n'existe pas de chevaux sont déviants dans la mesure où ils assertent « la non existence d'un être dont l'existence est déjà présupposée par l'emploi de cheval » (p. 90). Or, on obtient des tournures impersonnelles 
acceptables dès que le nom simple est remplacé par un SN complexe définissant un sousensemble d'objets, comme dans Il existe des chevaux à poil ras! Il n'existe pas de chevaux à poil ras. ( Ibid., pp. 93-94).

4. Cf. Babby (1980, p. 63): " An ADS [affirmative declarative sentence] normally presupposes its subject's existence and asserts that the subject performs the action denoted by the verb. In an NDS [negative declarative sentence] it is usually presupposed that the subject exists; it is asserted that this subject does not perform the action or is not in the state denoted by the verb. In other words, NDS's normally presuppose that the subject NP has a referent and assert that the action denoted by the verb is not realized. »

5. Cf. par exemple Picabia, 1987 : «La construction il y a SN est traditionnellement définie comme une construction existentielle ou présentative » (p. 236) ; « Il y a existentiel fonctionne comme il existe » (p. 241).

6. En russe, il s'agit de variations morphologiques (cf. Dahl, 1973, p. 81). En suédois, cette distinction surgit aux niveaux prosodique et lexico-syntaxique. Considérons à titre d'exemple la traduction de la phrase Aucune arme n'a été trouvée, qui est ambiguë en français. (a) correspond à son interprétation déclarative, (b) et (c) constituent des variantes correspondant à son interprétation existentielle. Notons que les majuscules signalent une proéminence prosodique.

a [SN INGET vapen] upphittades.

$\mathrm{b}$ [SN Inget VAPEN] upphittades.

c [SN Något VAPEN] upphittades inte.

(Inget est un quantificateur négatif, comme aucun; något correspond grosso modo à " quelque », inte signifie « ne... pas », vapen, « arme » et upphittades « a été trouvé ».)

7. Ce corpus, établi par Karl Johan Danell (département de langues romanes, Université d'Umeå), s'appelle CALIBSO. Au moment où je m'en suis servie, il se composait du texte intégral d'une trentaine de numéros des quotidiens belges et suisse Libre Belgique, Le Soir et Tribune de Genève, soit 1,5 millions de mots courants.

8. A côté de ces 498 occurrences, j'en ai relevé 202 intégrés dans un SPREP (dont 22 en fonction de complément d'objet indirect) et 26 dans un énoncé démuni de verbe.

9. Un facteur responsable de ce déséquilibre est la forte représentation du verbe avoir (38\%). En fait, une lecture référentielle d'un SN en AUCUN complément d'objet de avoir est difficilement concevable.

En position d'objet direct, AUCUN entre en concurrence avec PAS DE N, qui n'admet qu'une interprétation non référentielle (voir à ce sujet Heldner 1992, pp. 86-88). En tenant compte des 381 occurrences de PAS DE N compléments d'un verbe transitif, on constate que le nombre des SN objets non référentiels s'élève à 574 , soit 96 pour cent de l'ensemble des SN objets introduits par AUCUN ou PAS DE. Ce fait mériterait une discussion qui dépasse cependant le cadre de cet article.

10. Par énoncé j'entends ici le résultat d'une énonciation, autrement dit, un événement situé dans le temps et l'espace. Il s'agit donc d'un concept qui relève de la communication.

11. Il s'agit de P. Moinot, Le sable vif, 1963.

12. Cf. notamment Reboul 1988/1989 et 1989.

13. A ce sujet, voir par exemple Heldner, 1989, pp. 94-95.

14. D'après Babby (1980, p. 135), on a souvent fait cette observation à propos des verbes russes figurant dans un énoncé existentiel négatif. Dans la littérature, ceux-ci se décrivent comme " sémantiquement faibles ", " désémantisés " et même " redondants ».

15. Bien entendu, ce SN objet apparaîtra comme sujet à la suite d'une transformation passive.

16. Notons le parallélisme entre ces verbes et les verbes existentiels à cet égard.

17. Ainsi, par exemple, faire une étude correspond à étudier, prendre une décision à décider, avoir une crainte à craindre, etc. Dans certains cas on observe des «trous" tout à fait arbitraires dans le lexique. 
18. C'est probablement un effet du hasard que cela n'arrive pas avec la plupart des $\mathrm{N}$ susceptibles d'avoir un V support. Dans de nombreux cas, il y a des liens indirects, comme par exemple avec éprouver et crainte qui sont reliés par le terme définitoire "sentiment» sous leurs entrées respectives.

19. Cf. par exemple Mel'cuk \& Zolkovskij (1970).

20. Mathématiquement parlant, l'application d'une fonction à un argument ne peut donner qu'une seule valeur. Mais, dans ce contexte particulier, rien n'empêche, me semble-t-il, de faire donner plus d'une valeur par une seule fonction, tant que les valeurs obtenues synonymes, quitte à ne plus avoir affaire à une fonction au sens strict du terme. En ce qui concerne les verbes support non synonymes associés à un substantif donné, il faut envisager une succession d'applications de deux ou plusieurs fonctions.

21. "Les substantifs sous-catégorisés (- concret) ne s'assimilent pas aux substantifs syncatégorématiques, puisque l'on trouve des substantifs syncatégorématiques tels que course, tir, embarquement, dégel, etc., qui sont marqués du trait (+ concret).» Kleiber, 1981, p. 65.

\section{RÉSUMÉS}

Les syntagmes nominaux déterminés par l'indéfini AUCUN - que l'on peut envisager comme un quantificateur existentiel intégré dans le champ de la négation - reçoivent deux interprétations distinctes, surtout en position de sujet, suivant qu'ils apparaissent dans un énoncé déclaratif ou existentiel.

Une lecture non référentielle s'impose en proposition existentielle, où ces SN contribuent à asserter la non existence ou l'absence d'objets (ou de substances ou d'ensembles d'objets) de la catégorie désignées par le nom tête. L'énoncé Dans cette région, il n'y a aucune classe maternelle de plus de quatorze enfants en constitue un exemple caractéristique.

Dans des énoncés déclaratifs, comme Aucune maison n'a été épargnée, ce quantificateur négatif opère sur un sous-ensemble fini et spécifique d'objets de la catégorie dénotée par la tête nominale (c'est-à-dire maisons). L'existence de ce sous-ensemble étant présupposée, cette lecture est qualifiée de référentielle.

Le problème traité dans cet article est celui d'identifier les facteurs responsables de la sélection de l'une ou de l'autre lecture. En fait, il n'existe pas de critère formel simple permettant de décider si un énoncé donné doit s'analyser comme déclaratif ou existentiel, sauf quand celui-ci fait intervenir une construction impersonnelle comme il y $a$. L'approche adoptée ici consiste à explorer les possibilités d'une analyse purement sémantique cantonnée dans la phrase. Une éventuelle analyse pragmatique interviendra à un stade ultérieur pour rendre compte des cas demeurés ambigus au niveau phrastique. Un examen des données fournies par un corpus informatisé a donné les résultats suivants :

Un SN déterminé par AUCUN et sujet d'un verbe passif accepte seulement la lecture non référentielle, lorsqu'il a un nom tête syncatégorématique marqué [abstrait] et qu'il s'accompagne d'un verbe support. Quant aux SN en AUCUN sujets d'un verbe existentiel, ils manifestent une forte tendance à recevoir une interprétation non référentielle. En revanche, la lecture référentielle s'impose dès que le nom tête du SN sujet est catégorématique et marqué [animé] ou [concret] et que la structure sémantique du verbe est suffisamment riche pour lui assurer une certaine autonomie. En combinant un verbe qui impose peu de contraintes sémantiques à son sujet - ou aucune - à un SN sujet dénotant un référent concret ou animé, on obtiendra souvent 
une phase ambiguë, dont l'ambiguïté ne sera levée que par une prise en compte de facteurs discursifs ou pragmatiques.

This study is concerned with the interpretation of French noun phrases containing an instance of the indefinite determiner AUCUN, which may be seen as existential quantifier in the scope of negation. Such noun phrases give rise to two distinct readings according as they appear in existential or declarative sentences, particularly in the subject position.

In existential propositions an NP of the structure AUCUN N is used to asset the non existence of entities, substances, or sets of the category denoted by $\mathrm{N}$ relative to a specific location in time and space, as in Dans cette région, il n'y a aucune classe maternelle de plus de quatorze enfants. This reading of AUCUN $\mathrm{N}$ is being refused to as non referential.

In a declarative sentence like Aucune maison n'a été épargnée, the negative quantifier operates on a finite, specific subset of entities of the category $\mathrm{N}$ (i. e. maisons), the existence of which is presupposed. It is asserted that the action, process, or state denoted by the verb does not hold for any of the members of this subset. This reading is claimed to be referential.

The question addressed here is that of identifying the factors which determine the interpretation of an actual utterance. In fact, ther exist no simple formal criteria for deciding whether a given utterance containing a noun phrase of this structure should be analysed as declarative rather than existential, except where an explicit use of an impersonal construction such as il ya is involved. This problem must clearly be solved in order for a parser to work out valid inferences from this type of sentences. My approach is exclusively semantic, but an analysis along pragmatic lines may prove necessary in the end to account for certain cases. An analysis of the data obtained from a computer readable corpus yields the following results.

A non referential reading is alone available for a subject NP of the type AUCUN N inpassivized sentences, if its head noun features the properties abstract and syncategorematic, and if it takes a verb which typically collocates with this noun (a «support " verb). Furthermore, there is a strong tendency for any subject NP containing AUCUN to be interpreted non referentially when combined with an intransitive existential verb, although a referential reading is shown not to be entirely excluded. On the other hand, the referential reading obtains whenever the nominal head of the subject NP is categorematic and subcategorized animate or concrete and combines with a verb whose semantic structure is sufficiently rich to gurarantee its autonomy. The combination of a verb, which imposes few semantic constraints on its subject - or none - and a subject NP denoting a concrete or animate referent, tends to produce an ambiguous sentence, which may only be disambiguated by taking pragmatic factors into account.

\section{AUTEUR}

\section{CHRISTINA HELDNER}

Institut d'Etudes romanes, Université de Göteborg 